\title{
Stigmata des Krieges in der Literatur des geteilten Korea*
}

\author{
Sam-Huan Ahn (Seoul National Uni)
}

Das Land Korea wurde, nachdem es 1945 vom japanischen Joch befreit worden war, mit dem Beginn des Kalten Kriegs von den beiden Großmächten USA und UdSSR in zwei Teile, in Nord- und Südkorea geteilt. Diese Teilung wurde weder nach irgendwelchen ethnischen Logiken geschweige denn nach dem Willen der Bevölkerung des Nordens und des Südens vollzogen. Vor der Teilung hatte es nicht einmal differenzierte geographische oder ethnologische Unterschiede zwischen Nord- und Südkorea gegeben. Die koreanische Halbinsel wurde einfach willkürlich und brutal am 38. Breitengrad in zwei Hälften geteilt, als ob die kleine Halbinsel, auf der seit der alten Zeit ein Frieden liebendes Kulturvolk lebte, ein Stück Kriegsbeute wäre. Wir wissen, die Geschichte kennt eher selten die Kategorie Gerechtigkeit. Denn es wurde ja nicht etwa die japanische Insel Hokkaido geteilt, sondern die koreanische Halbinsel, als ob die Koreaner irgendeine Mitschuld am Zweiten Weltkrieg gehabt hätten. Wie paradox dies aus historischer Perspektiv ist, kann man sich lebhaft durch das Gedankenspiel vor Augen führen, dass die vier Siegermächte nicht etwa das besiegte Deutschland geteilt hätten, sondern etwa das schuldlose Polen.

Diese Betrachtungen über die Willkürlichkeit der Geschichte schicke ich voran, weil ich unterstreichen möchte, dass die Koreaner nicht etwa ihrem

\footnotetext{
* Dieser Beitrag wurde ursprünglich verfasst als Vortrag, den ich als einen der „Tübingen Korean Studies Lectures“ am 18. Juni 2014 an der Universität Tübingen gehalten habe. Er ist für den Druck nur geringfügig verändert worden.
} 
Naturell nach „feindliche Brüder“ sind, sondern erst infolge der Kontingenzen des Zweiten Weltkrieges und des „Kalten Kriegs“ so zu „feindlichen Brüdern“ wurden. Die zwei erzfeindlichen Fronten auf der koreanischen Halbinsel sind nicht nur das Produkt der Politik von RHEE Syngman und KIM Il-Sung, sondern auch ein Ergebnis des weltweiten Kalten Krieges. Der Korea-Krieg der Jahre 1950-1953 war in gewissem Sinne ein Stellvertreterkrieg der beiden Großmächte des Kalten Krieges, dessen Folgen das koreanische Volk zu erleiden hatte.

\section{Krieg als Wunde des Menschen überall und immer wieder}

Der Korea-Krieg ist in der modernen südkoreanischen Literatur sehr präsent. (Hier rede ich leider nur von Südkorea, da man über Nordkorea und die nordkoreanische Literatur überhaupt wenig weiss.)

Das erste, bedeutendste literarische Werk über die Teilung Koreas ist wohl der Roman von CHOE In-Hun (geb. 1936), „Der Platz“ (1960). YI Myeong-Jun, ein Akademiker, der an einer Elite-Universität in Seoul Philosophie studiert hat, wird eines Tages ganz überrascht zur Polizei vorgeladen und zur Rede und Antwort gestellt, ob er noch in Kontakt wäre mit seinem Vater, der nach der Teilung Koreas nach Nordkorea gegangen war und dort gewisse führende Position innehatte. Trotz seiner Verneinung musste er Gewalttat des Polizisten erleiden. Die Polizisten, die unter der japanischen Herrschaft die patriotischen Bürger und Intelligenten von damals schikanierten und folterten, sind auch in der neuen Republik Korea dieselben geblieben. Aber auch im Norden Koreas, wohin er nach der tiefen Frustration 
im Süden durch eine illegale Schiffahrt geflüchtet war, musste er enttäuscht werden. Denn da in der Demokratischen Volksrepublik Korea musste er „die stolze Leidenschaft im Herzen “1) des Menschen vermissen. Nach seiner Meinung gebe es im Norden nur noch den angeblich gemeinsamen „Platz“ ohne den menschlichen „Privatraum“, während es im Süden nur den individuellen „Privatraum“ ohne irgendeinen sinnvollen gesellschaftlichen „Platz“ gibt. Schliesslich muss er sich als Kriegsgefangener vom südkoreanischen Gefangenenlager weder Nordkorea noch Südkorea, sondern ein Drittes Land wählen, um inmitten der Schiffahrt nach Indien verschollen zu werden. Für den Leser dieses Romans bleibt schliesslich keine Hoffnung mehr, dass diese tragische Figur Yi noch auf dieser Welt lebt.

Aber auch in harmlosen Erzählungen Südkoreas kann eine Kriegssituation überraschend wie ein Gespenst auftauchen, oder sie wird wie eine Wunde der literarischen Figuren nur beiläufig erwähnt. Hiervon gibt es beliebig viele Beispiele. Um hier nur ein Beispiel zu geben, sei eine Stelle von der Novelle des Schriftstellers HYEON Gil-Eon (geb. 1940), „Auf dem Weg nach der Insel“, zitiert, in der ein Bruchstück des Krieges anscheinend ohne notwendigen Grund so kurz erwähnt wird wie folgt:

Die Dämmerung nach dem Sonnenuntergang ist schön, aber da diese Dämmerung die Dunkelheit nach sich herbeiführt, ist sie auch zu fürchten. Noch erinnere ich mich an das Schicksal meiner Mutter, die leben musste, indem sie auf diese Dunkelheit wartete. Denn mein Vater, der als Partisan in die Berge gegangen war, kam erst in der stockdunklen Nacht der letzten Tage des Monats nach dem Mondkalendar ins Dorf geschlichen, um an das Fenster des Hinterzimmers, wo seine Braut wohnte, leise zu klopfen. Noch im dritten Monat seit der Heirat war der Bräutigam in die Berge geflüchtet, um nicht von der Polizei verhaftet zu

1) In-Hun Choe: Der Platz, S. 133, in: In-Hun Choe: Sämtliche Werke, Munhakgwajiseongsa 2010, Bd. 1, S. 23-209. 
werden, und so wurde er Partisan.

Seine Eltern wussten zwar genau, was da im Zimmer ihrer Schwiegertochter passierte, aber sie stellten sich, als ob sie nichts davon wüssten. Meine Mutter hätte kaum schlafen können, wenn sie in der tief dunklen Nacht draußen irgendwo im näheren Dorf etwa ein Hund heulen hörte. Erst als ich im Begriff war, meine Heimat zu verlassen, bekam ich von diesen Stunden in der Dunkelheit hören, in denen die Braut, meine Mutter, auf ihren Bräutigam wartete. Damals konnte ich mich noch nicht voll in die Lage meiner Mutter einfühlen, in deren Herz sich die Verzweiflung, das Warten und die Vorfreude auf das gefährliche Treffen in der stockdunklen Nacht ohne den Mond am Himmel gekreuzt haben dürften.2)

Hier handelt es sich eigentlich nicht um den Korea-Krieg selbst, sondern um den 4.3-Aufstandes, der als eine Vorform des Korea-Kriegs zwischen den südkoreanischen Soldaten und Polizisten unter der amerikanisch-militärischen Regierung und den südkoreanischen Kommunisten am 3. April 1948 in der Insel Jeju ereignet hat. Inmitten dieses Konflikts wurden viele schuldlose Zivilbevölkerung von der Jeju-Insel als Kommunisten oder deren Mitläufer verfolgt bzw. ermordet. Jedenfalls kann man hier wohl nicht festlegen, dass diese Erinnerung und Erwähnung des Ich-Erzählers „ohne notwendigen Grund“ wäre. Sicher hätte auch diese Stelle ein Recht, sich erzähltechnisch genau dort zu behaupten. Aber was ich hier zeigen möchte, ist die Tatsache, dass der Kampf zwischen den Rechten und den Linken wie ein dunkler Schatten und wie ein Gespenst jederzeit und überall in der gegenwärtigen koreanischen Literatur erwähnt zu werden pflegt. Denn es ist eben diejenige Wunde der Koreaner, die der Konflikt zwischen den Rechten und den Linken seit dem Jahr 1945 hinterlassen hat und die noch lange nicht ganz geheilt worden ist. Aus der obigen kurzen Erwähnung des Schicksals seiner Mutter

2) Gil-Eon Hyeon: Auf dem Weg nach der Insel, S. 159, in: Kann nicht ein jeder zu der Insel gehen?. Erzählungen, Seoul: Mulle 2014, S. 111-205. 
kann man schon herleiten bzw. vermuten, was für ein saueres Leben auch dieser Ich-Erzähler inzwischen hinter sich zu bringen hatte. Denn die Lebensbedingungen der Eltern können ja, wie diejenigen des Vaters von YI Myeong-Jun, auch und besonders im geteilten Korea den Lebenslauf des Sohns stark beeinflussen.

\section{Massaker und Ermordung als literarischer Alltag}

Selbstverständlich erscheint der Krieg in der koreanischen Literatur nicht immer so harmlos wie in unserem obigen Beispiel, in dem seine Schrecken nur nebenbei erwähnt werden. Wie in der Literatur anderer Völker, die den Krieg erlitten haben, wird auch der Korea-Krieg vor allem als inhumanes und brutales Massaker an der unschuldigen Zivilbevölkerung dargestellt. In der modernen südkoreanischen Literatur seit den 1950er Jahren erscheint der Bericht über den Massenmord ebenso häufig wie normal. Noch im Roman von GIM Won-Il (geb. 1942), „Der Vater des Sohns“ (2013), wird folgendes berichtet:

Etwa nach 10 Tagen nach dem Ausbruch des Korea-Krieges am 25. Juni 1950, also vom 4. Juli 1950 an, begannen die Sondertruppe des koreanischen Heers (CIC, Counter Intelligence Corps) und die Militärpolizei die Häftlinge und die Inhaftierten im Daejeon-Gefängnis einschließlich vieler Zivilbevölkerung, die früher gegen die amerikanische Militärregierung oder gegen die Syngman-Rhee-Regierung gewesen waren, kollektiv hinzurichten. Der Justizbeamte der amerikanischen Armee, Oberstleutnant Edward, der am Ort des Massakers anwesend gewesen war und die Hinrichtungsszenen als Photos hinterließ, zeichnete auf: „Es brauchte drei Tage, bis man 1,800 politische Verbrecher 
hinrichtete. Es geschah in der ersten Woche des Julis 1950.“ Anschließend massakrierten die Sondertruppe und andere militärisch-polizeiliche Truppen mehr als 7,000 linke Inhaftierte einschließlich der Teilnehmer am Bund der Demokratisch-Bekehrten in mehreren Tagen massenweise. Man hatte die Inhaftierten aus dem Gefängnis getrieben und die Lastwagen mit ihnen voll beladen, um sie dann auf den Hinterberg des Mangwol-Dorfes, Sannae-myeon, Daedeok-gun [in der Nähe der Stadt Daejeon - S. H. Ahn] zu bringen. Dann befahl man ihnen, sich vor den schon vorher gegrabenen Gruben auf dem Bauch zu legen, so dass man sie von hinten in den Rücken schoß. Jeden Tag brachte man von fünf bis fünfzehn Lastwagen voll Menschen dorthin, und sie alle richtete man in mehreren Tagen hin. Diese Opfer bestanden größten Teils aus ehemaligen Mitgliedern der Südkoreanischen Arbeiterpartei, aus den Partisanen, die inhaftiert worden waren oder sich selbst schon vor dem Krieg der Polizei ausgeliefert hatten, oder den Teilnehmern am Bund der Demokratisch-Bekkehrten. Im Großen und Ganzen waren sie Bauern, und unter denen gab es auch viele Studenten.“3)

Der Erzähler dieses Romans von GIM Won-Il berichtet weiter, dass in der Zeit des weiteren Rückzugsgefechts auch in Gyeongsan viele politisch links stehende Inhaftierte des Daegu-Gefängnisses und dieser Region massakriert wurden; in Masan wurde 1,681 Menschen einschließlich der politischen Häftlinge des Masan-Gefängnisses hingerichtet, während 750 Menschen in Gimhae und Euichang, 870 in Ulsan, 712 in Yangsan und 150 in Changnyeong ermordet wurden.

Dieses kollektive Massaker beging die südkoreanische Regierung, da sie fürchtete, dass sich diese linken Häftlinge und auch die vorher schon zur Demokratie Bekehrten eventuell der siegreich südwärts vordringenden Volksarmee anschließen würden.

3) Won-Il Gim: Der Vater des Sohnes. Die Zeit des Vaters und die Kindheit des Sohns, Seoul: Munhakgwasasangsa 2013, S. $305 f$. 
Brutal ermordet werden die unschuldigen Menschen auch im Roman von HWANG Sok-yong (geb. 1943), „Der Gast“ (2007). Der Pfarrer RYU Yoseop sagt es so in seinem Gebet anlässlich eines Hausgottesdienstes bei seinem älteren Bruder Yohan in einer Weißensiedlung von New Jersey, USA:

\begin{abstract}
Vor vierzig Jahren sind wir aus unserer Heimat fortgegangen. [ ... ] Unser Land befand sich im Krieg. Viele unschuldige Menschen kamen ums Leben. Man brachte sich gegenseitig um, nur um zu überleben. [ ... ] Wir müssen zuerst Buße tun.4)
\end{abstract}

Erst als sein Bruder Yoseop weg war, fielen dem allein gebliebenen Yohan auf einmal die toten Menschen aus seinem Heimatdorf Chansaemgol in Nordkorea ein. Es waren all die Geister derer, die er in seiner von der kommunistischen Herrschaft wieder befreiten Heimat ohne Mitleid und Nachsicht ermordet hatte. Die Gestalt des Il-Lang, des ehemaligen Bauernknechts seines Hauses, sah er auch vor sich. Il-Lang hatte damals unter der kommunistischen Herrschaft als Vorsitzender des Dorfkomitees seiner Familie das Land weggenommen, und ihn ermordete Yohan kurz vor dem Einmarschieren der amerikanisch-südkoreanischen Truppe dann brutal. Unter den Gestalten befanden sich auch Chungsons Frau, die Volksschullehrerin und die sechs kleinen Schwester von Myeong-Seon also, auch Frauen, die er damals brutal ermordet hatte. Besonders rücksichtlos und erbarmungslos hatte er die zwei Mädchen mit den Musikinstrumenten mit dem kurzen Pagenschnitt ermordet, nur weil sie in der Uniform der Volksarmee gewesen waren. Offenbar gehörten sie zu einer Gruppe, die die Soldaten der Volksarmee mit ihren Konzerten aufmuntern sollte. Der kleine Yoseop hatte ihnen in ihrem Versteck Reis und andere Speisen gebracht. Als

4) Sok-yong Hwang: Der Gast. Roman, Aus dem Koreanischen von Young Lie, Katrin Mensing und Matthias Augustin, München: Deutscher Taschenbuch Verlag 2007, S. 17. 
Yohan diese „Kommunistinnen“ entdeckte, ermordete er sie brutal, was damals die zarte Neigung des kleinen Knabens in der Pubertät, also seines kleinen Bruders Yoseop, erschüttert haben musste.

Durch den Durst wieder aufgeweckt, sah Yohan in seinem Wohnzimmer, dass jemand auf dem Sofa saß.

\section{Wer ist da?}

Das schwarze Ding antwortete mit heiserer, tiefer Stimme. Ich bin's. Erkennst Du mich nicht?

Wer du bist, hab’ ich gefragt!

Das Ding sagte mit einer Stimme wie voller Kohlenstaub: Der Maulwurf aus dem Bergwerk von Eunyul.

Sunnam? Du?

In dem Moment vergaß ich [Yohan - S. H. Ahn] alles und schaltete freudig das Wohnzimmerlicht an. Nur die Möbel und der Fernseher standen, wo sie immer standen [ ... ], und mit einem Mal wich alle Kraft aus meinen Beinen. Sunnam war ungefähr zehn Jahre älter als ich, also damals schon um die Fünfunddreißig. [ ... ] Ich erledigte ihn im Winter jenes Jahres. Am Strommast, der an der Stelle stand, wo die neue Straße von Chansaemgol zur Kreisstadt und der Feldweg sich trafen, knüpfte ich Sunnam mit einer Drahtschlinge auf.5)

Es ist hier bezeichnend, dass Yohan aktuell alles, was früher passierte, vergisst und vor Freude am Wiedersehen mit Sunnam das Wohnzimmerlicht anschaltet.

Dieser alte Geisterseher schrie aber noch am nächsten Tag zu seinem Bruder Yoseop, der ihm telephonisch vorschlägt, zu Gott um Vergebung zu beten, damit auch die Toten in Frieden ihre Augen schließen: „Warum soll ich um Vergebung betteln? Die Roten sind Söhne des Teufels! Satansbande! Ich stehe auf der Seite von Erzengel Michael, und die, das sind die Bestien

5) S. Y. Hwang: Der Gast, S. 22f. 
aus der Apokalypse! Wenn unser Gott es mir befiehlt, würde ich auch heute noch gegen diese Teufel kämpfen!“6)

Gleich am selben Abend starb Yohan. Sein Bruder Yoseop, der damals im nordkoreanischen Heimatdorf Chansaemgol altersgemäß an den vielen Mordtaten seines älteren Bruders wenig mitschuldig war, flog nach Yohans Bestattung als einer der amerikanisch-nordkoreanischen Heimatbesucher mit einem Stück Knochen von der Asche seines Bruders nach Pyeongyang, um es dann in seinem Heimatdorf begraben zu können.

Wie hieraus zu ersehen ist, entstand der Konflikt im Norden Koreas meist zwischen Protestanten und Kommunisten, während es sich im Süden eher um das Massaker der Zivilbevölkerung durch das Militär oder die Polizei handelte.

Aber im Grunde wurde diese Tragödie sowohl im Norden als auch im Süden nach der Meinung des Schriftstellers Hwang verursacht - von der Krankheit aus dem Westen, wie die Pocken, die früher einmal „im Volksmund Gast genannt wurden [ ... ]. Bei mir [S. Y. Hwang] steht die Bezeichnung Gast allerdings auch für zwei andere Dings: das Christentum und den Marxismus."7)

Ob Krieg zwischen Christentum und Marxismus oder Demokratie und Kommunismus - die Koreaner kämpften wegen dieser beiden westlichen Gäste auf Leben und Tod, und viele unschuldige Menschen kamen dabei ums Leben.

6) S. Y. Hwang: Der Gast, S. 25.

7) S. Y. Hwang: Der Gast, S. 296 (Nachwort des Schriftstellers Hwang Sok-yong). 


\section{Die Selbstzensur und die unsichtbare Bedrohung der Feindbegünstigung}

Da es keinen Sinn mehr macht, weitere Beispiele von Massakern und Morden der „feindlichen Brüder“ bloß aneinander zu reihen, möchte ich hier lieber auf andere Stigmata des Kriegs in der Literatur des geteilten Landes Korea hinweisen.

Zunächst erlaube ich mir hier aber, eine Stelle aus der DDR-Literatur zu zitieren:

Er [Eumelos] zog die Schrauben an. Er warf sein Sicherheitsnetz, das bisher die Mitglieder des Königshauses und die Beamtenschaft gedrosselt hatte, über ganz Troia, es betraf nun jedermann. Die Zitadelle nach Einbruch der Dunkelheit gesperrt. Strenge Kontrollen alles dessen, was einer bei sich führte, wann immer Eumelos dies für geboten hielt. Sonderbefugnisse für die Kontrollorgane.8)

Ich glaube, dem deutschen Publikum käme dieses Zitat bekannt und vertraut vor. Es ist eine Stelle aus Christa Wolfs Erzählung „Kassandra“, die den antiken Mythos von dem Kriegszustand zwischen den Trojanern und den Griechen auf die Zeit der 80er Jahre des letzten Jahrhunderts zwischen den beiden Blöcken des Kalten Krieges zu übertragen versucht. Davon lesen wir ein bisschen weiter:

Was kommen mußte, kannte ich schon, den festen Achselgriff, die Männerhände, die mich packten, das Klirren von Metall auf Metall, der Geruch von Schweiß und Leder. [ $\cdots$ ] Ich erfuhr, wie eine Gefangene die Zitadelle von Troia sieht, befahl mir, es nicht zu vergessen. Vergaß es nicht, doch hab ich an den Weg

8) Christa Wolf: Kassandra. Erzählung, Darmstadt und Neuwied 1983, S. 116. 
unendlich lange nicht mehr gedacht. Warum. Mag sein, der halbbewußten Schläue wegen, deren ich mich schämte. Denn warum schrie ich, wenn ich schrie: Wir sind verloren, warum nicht: Troer, es gibt keine Helena! Ich weiß es, wußte es auch damals schon: Der Eumelos in mir verbot es mir.9)

Diese Lage von Kassandra kennen auch die koreanischen Dichter. Sie kennen auch bei sich die „halbbewußte Schläue“, mit der sie beim Schreiben eine Art „Selbstzensur“ vornehmen müssen. „Der Eumelos in mir“ - es ist dies, was nicht nur bei Kassandra, sondern auch bei manchem koreanischen Dichter während der Diktatur (z. T. auch noch in der Gegenwart) Koreas zu finden ist.

Da sprach der Vater, der bis jetzt geschwiegen hatte: Schweig, Kassandra.

Zornig, böse. - Ich sagte: Vater - Komm mir nicht mehr mit „Vater“. [ ‥] Ja kennst du unsere Lage überhaupt? Und wenn du diesem unsern Plan, Achill, den schlimmsten Feind zu töten, jetzt nicht zustimmst - weißt du, wie ich das nenne? Feindbegünstigung. ${ }^{10)}$

Wieso ist dieses jedem schreibenden Südkoreaner (dem Nordkoreaner wohl noch mehr?) zum Schrecken bekannte Wort, „Feindbegünstigung“, hier auch im Werk von Christa Wolf zu lesen? Ist es nicht frappierend, dass das Wort „Feindbegünstigung“, diese unsichtbare Kette für jeden koreanischen Schriftsteller, auch in der Literatur der DDR vorkommt?

Diese Frage ist insofern leicht beantwortet, als auch Christa Wolf feindliche Fronten darstellt, nämlich die Trojaner und die Griechen. Auch Christa Wolf spricht so im übertragenen Sinn von den feindlichen Fronten des Kalten Krieges, sie erzählt ja von der Konfrontation von Ost und West.

9) Chr. Wolf: Kassandra, S. 79.

10) Chr. Wolf: Kassandra, S. 143. 
Der Vergleich mit Christa Wolf hat es plausibel scheinen lassen, dass auch die koreanische Literatur das Phänomen Selbstzensur und die Angst vor „Feindbegünstigung“ thematisiert.

Angesichts der Konfrontation mit dem Norden bekamen viele koreanische Dichter ab den 1950er Jahren bis zur Gegenwart Angst vor diffamierenden Beschuldigungen, und sie erlegten sich, bewusst oder nicht, eine Art Selbstzensur auf. Angesichts der Konfrontation an der DMZ, der Demilitarisierten Zone, könnte selbst konstruktive Kritik an der Regierung wenn nicht als Verschwörung, so doch als Angriff gegen den Staat, als ein Akt der „Feindbegünstigung“ eben, diffamiert werden.

Um diese Aspekte der koreanischen Literatur zu veranschaulichen, sei hier eine Stelle der Erzählung „Die Mauer der Gerüchte“ (1971) von YI Cheong-Jun (1939-2008) herangezogen:

Nach dem Ausbruch des Korea-Kriegs am 25. Juni 1950 pflegten in unserem Heimatdorf nachts die Polizisten und die Partisanen wechselweise zu besuchen. Eines Nachts drangen schon wieder Leute ins Dorf ein, die nicht leicht $\mathrm{zu}$ identifizieren waren, ob sie Polizisten oder Partisanen waren. Und einer von denen kam auch zu unserem Haus, und plötzlich raffte er die Tür unseres Zimmers auseinander, wo Mutter und ich schliefen. Unter dem blendenden Licht einer Taschenlampe dicht am Gesicht meiner Mutter fragte er sie, an welcher Seite sie stand. Aber meine Mutter konnte in dem Augenblick diese Frage nicht sogleich beantworten. Denn sie konnte ja nicht gleich unterscheiden, wer hinter dem grellen Licht der Taschenlampe war, ob er ein Polizist oder ein Partisan war. Es war ja klar, es würde sich rächen, wenn sie falsch antworten würde. Dennoch war meine Mutter gezwungen, da doch zu antworten, ohne dass sie wusste, wer ihr Gegenüber war. Ihre Lage war verzweifelt. Heute noch erinnere ich mich genau und lebendig, wie es mir in jenem verzweifelten Augenblick zumute war und wie furchtbar es uns war - vor dem grellen Licht vor uns.11)

11) Cheong-Jun Yi: Sämtliche Werke, Bd. 4: Die Mauer der Gerüchte, Seoul: 
Dies ist die Schilderung einer typischen Szene, die während des Korea-Kriegs in entlegenen Dörfern fast alltäglich war. Yi hat aus diesem Trauma seiner Kindheit allerdings ein psychoterapeutisches Symptom seiner Figur, eines problematischen Schriftstellers im Südkorea der 1970er Jahre gestaltet, jener Zeit also, in der die Militärdiktatur die Freiheit der Schriftsteller unter dem Vorwand der Erhaltung des demokratischen Systems der Republik Korea einzuschränken begann.

Diese Schriftsteller-Figur in der Erzählung, BAK Jun, schrieb selber mehrere Novellen, genauer gesagt: Novellen in der Rahmenerzählung von Yi. In einer dieser Texte wird der Schriftsteller G., also die Schriftsteller-Figur von BAK Jun, von der Phantasie verfolgt, dass er, ähnlich wie Josef K. in Franz Kafkas Roman „Der Prozess“, verhaftet und von einem Prüfer zum Verhör geladen wird:

Aber G. musste zögern. Er konnte nicht wissen, wer der Prüfer sei. Dieser hatte eine Uniform an, die G. niemals gesehen hatte. [ $\cdots$ ] Dass man einem Gegenüber, dessen Identität einem nicht bekannt ist, das Wahrste über sich selbst auszusagen hat, macht einen schon unruhig. ${ }^{12)}$

Schließlich sagt ihm der Prüfer, „Ihre Furcht vor dem Licht der Taschenlampe und vor mir, das ist schon das Leiden Ihrer Strafe, das Sie sich selbst gewählt haben. Und es ist sicher, dass Sie mit diesem Leiden schon psychisch halb krank sind, oder bald krank werden. Vor unserem Urteil büßen Sie schon von sich aus Ihre Strafe......"13)

Erst hier wird uns klar, warum sich der Schriftsteller BAK Jun selbst als einen Wahnsinnigen ins psychiatrische Krankenhaus eingeliefert hat. Er leidet

unhakgwajiseongsa 2011, S. 219.

12) C. J. Yi: Die Mauer der Gerüchte, S. $226 f$.

13) C. J. Yi: Die Mauer der Gerüchte, S. 238. 
nämlich als gewissenhafter Schriftsteller seiner Zeit unter der Zensur und Selbstzensur: BAK Juns und G.s Angst vor dem grellen Licht der Taschenlampe ist nichts anderes als die Angst vor einem Unbekannten ohne Identität, nämlich vor der „Mauer der Gerüchte“. Bak selbst spricht ja in der Erzählung davon, „dass sein Gegenüber in der Bekleidung eines Gerüchtes erscheint, um seine Identität nicht zu enthüllen.“14)

Yis Erzählung, „Die Mauer der Gerüchte“, handelt also nicht vom Korea-Krieg selbst, sondern von einer der vielen Folgeerscheinungen dieses Brüder-Krieges, nämlich vom unsichtbaren System der Zensur und Selbstzensur und der Einschränkung der Freiheit des Schriftstellers. Indem Yi in diesem Werk mehrere Dimensionen und Ebenen vielschichtig darstellt, gelingt es ihm, den Krieg und dessen langwierigen negativen Nachwirkungen für die koreanische Gesellschaft zur Gestaltung zu bringen.

GIM Won-Ils Roman, „Blaue Seelen“ (2005), besteht eigentlich aus sechs seriellen Erzählungen. Er behandelt das Leben und den Tod von acht Menschen, jenen acht Opfern des 9. Aprils 1975, der als „Tag der Schande der Justiz“ von der Internationalen Juristenkommission in Genf bezeichnet wurde: Er handelt von den acht Häftlingen, SEO Do-Won, DO Ye-Jong, SONG Sang-Jin, UH Hong-Seon, HA Jae-Wan, YI Su-Byeong, GIM Yong-Won und YEO Jeong-Nam, die als angebliche „Untergrundaktivisten der Volksrevolutionspartei“ wegen angeblicher „Verschwörung gegen die Republik Korea“ von der südkoreanischen Justiz zu Tode verurteilt wurden.

„Wieviel Uhr ist es? In dieser Nachtsstunde? Was ist los?“ fragte DO [ Ye-Jong ], indem er heftig hustete.

„Vier Uhr fünfundzwanzig.“ Sobald der Beamte vor ihm so antwortete, fesselten ihn andere zwei Beamte neben ihm mit doppelten Strängen und spannten es ihm

14) C. J. Yi: Die Mauer der Gerüchte, S. 250. 
noch fester an. [ ... ]

„Warum so abrupt? Darf ich den Grund wissen?“ [ ... ]

„Lassen wir uns doch ausgehen! Bitte kein großes Aufsehen und seien Sie doch ruhig! Es kann vielleicht eine gute Nachricht in diesem frühen Morgen geben!“"15)

So holte man einen nach dem anderen der acht unschuldig Verurteilten in der Morgenfrühe des 9. April 1975 aus ihrer Zelle. Es gab aber keine „gute Nachricht“, etwa dass man den Häftlingen ein Gespräch mit ihren Angehörigen erlaubt hätte, sondern man richtete sie binnen 18 Stunden nach ihrer Verurteilung hin. „Amnesty International“, „Die Vollversammlung der Koreanischen Staatsbürger für die Wiederherstellung der Demokratie“ und „Die Vereinigung der Katholischen Pfarrer für die Verwirklichung der Gerechtigkeit in der Koreanischen Gesellschaft“ protestierten am 10. April 1975 gegen die irrationalistische Barbarei des diktatorischen Regimes.

Erst 30 Jahre nach dem Jutizmord, also 2005, konnte Gim Won-Il das Leben und den Tod der Opfer als literarisches Werk unter dem Titel „Blaue Seelen“ publizieren. Und erst nach 32 Jahren, im Januar 2007, wurden die Opfer von der koreanischen Justiz als unschuldig erklärt und rehabilitiert. Wie erwartet hatte es sich als falsch erwiesen, dass es eine „Volksrevolutionspartei“ überhaupt gegeben hatte.

Dieses Werk von GIM Won-Il ist nicht nur als „verspätete“ Auseinandersetzung mit der Landesteilung nach dem Korea-Krieg beachtenswert, sondern er veranschaulicht auch auf bemerkenswerte Weise den Entstehungsprozess der „Gerüchte“ im Sinne der zuvor dargestellten Erzählung „Die Mauer der Gerüchte“ (1971) von YI Cheong-Jun.

Durch den Vergleich wird auch nochmals deutlicher, was YI Cheong-Jun mit der „Mauer der Gerüchte“ gestalten wollte und worauf er überhaupt

15) Won-Il Gim: Blaue Seelen. Ein Roman aus sechs Serien-Erzählungen, Seoul: Irum, 2005, S. 303f. 
anspielte. Denn tatsächlich fälschte die Diktatur auch vier Jahre nach dem Erscheinen von der „Mauer der Gerüchte“ noch die Fakten in der Affäre um die fiktive „Volksrevolutionspartei“ und die angebliche „Verschwörung gegen die Republik Korea“. Das Ziel davon war es, die Intellektuellen in Korea einzuschüchtern und vor eventuellen Widerständen gegen das Regime abzuschrecken.

\section{Das Schreiben in Korea}

Bisher habe ich über die versteckte, doch überall und jederzeit latent vorhandene Gefahr des Missbrauchs angeblicher „Feindbegünstigung“ sowie über die unsichtbare, aber gleichfalls allgegenwärtige Wirkung der „Zensur“ bzw. „Selbstzensur“ in der modernen koreanischen Literatur gesprochen.

Allerdings hat die koreanische Literatur aber auch einen mutigen, niemals konformbereiten Dichter wie GIM Nam-Ju (1946-1994) hervorgebracht.

Ein kleines Stück Stein

Zwischen Himmel und Erde

Wehte es kaum ein Zug Wind - bedrückend und beklommen!

An dem Tag, wo es mich beinahe erstickte und mein Herz vor Trauer zerbarst,

Gingen mein Freund und ich auf dem Damm spazieren,

Und wir wollten ein kleines Stück Stein sein,

Der aufs Wasser des Flusses

Eine kleine Welle zeichnet

Und bald versinken wird.

So ein kleines Stück Stein. 
Der Tag war vorbei und es wurde tief-schwarze Nacht!

Mein Freund und ich gingen den nächtlichen Weg,

Und wir wollten ein Zündfunke sein,

Der etwa wie ein Glühwurm auf dem Grasfeld schimmert

Und bald verschwinden wird, wenn der neue Tag kommt.

So ein Zündfunke.

Damal habe ich meinen Freund nicht gefragt,

wie schwer das kleine Stück Stein für die Geschichte wäre.

Damals habe ich meinen Freund nicht gefragt,

wie weit der Zündfunke den Raum der Dunkelheit vertreibt.

Nur war ich so stolz darauf, einen Freund neben mir zu haben,

Der bereit war, mit mir gemeinsam zu sterben.16)

Dieses Gedicht von GIM Nam-Ju zeugt nicht nur von großem Mut, sondern auch von literarischem Niveau. Es kennt etwa auch die Wirkung des Symbols: „Das Licht bei ihm ist die helle Welt, die neue Welt, wo alle ohne Unterdrückung leben können, während die Dunkelheit die finstere Welt, die zu erneuernde Welt ist, wo man das Volk unterdrückt und dieses dann nicht mehr im Stande ist, menschlich zu leben.“17) Das lyrische lch und sein Freund wollten „ein Zündfunke“ sein, „der etwa wie ein Glühwurm auf dem Grasfeld schimmert / Und bald verschwinden wird, wenn der neue Tag kommt.“ Hier fungieren „Zündfunke“ und „Glühwurm“ als eine Metapher für die neue Welt, während die „Dunkelheit“ eine Metapher für die politisch unterdrückte Gesellschaft ist.

So ist der Dichter GIM Nam-Ju fast ein einmaliges Phänomen in der

16) Nam-Ju Gim: Behausungsort des Gedankens. Gedichte, Seoul: Changjakgwabipyeongsa 1991, S. $26 f$.

17) Sungja Han: Metapher und Symbol in den Gedichten von Kim Nam-Ju, S. 243, in: Yom Mu-Ung / Lim Hong-Bae (Hrsgg.): Die Welt der Literatur von Kim Nam-Ju, Seoul: Changbi 2014, S. 235-246. 
modernen koreanischen Literatur: Er fürchtete kaum die Zensur, es schien, als ob er keine Selbstzensur gekannt hätte. Im Umfeld der „Demokratischen Bewegung in Gwangju“(1980) schrieb er ebenso mutige wie anspruchsvolle Gedichte. Unter dem Verdacht der „Feindbegünstigung“ musste er elf Jahre lang (1973 und dann von 1979 bis zu 1988) im Gefängnis einsitzen. Nach der Freilassung lebte er nur noch sechs Jahre. Es scheint, als ob er eines natürlichen Todes gestorben hätte, aber heutzutage stirbt ein normaler Koreaner selten mit dem Alter achtundvierzig. Jedenfalls ist sein Schicksal ein tragischer Nachhall der „Irrungen und Wirrungen“ der feindlichen Brüder Korea im 20. Jahrhundert.

Im Februar 2014 fand anlässlich seines 20-jährigen Todestages das Jubiläumssymposium „Wir denken an GIM Nam-Ju“ in Seoul statt. Ja, man müsste wohl wieder intensiver an GIM erinnern, da „in der koreanischen gegewärtigen Realität die demokratischen Werte, welche die Koreaner in den letzten Jahrzehnten mühselig errungen haben, total herausgefordert werden“18), wie es LIM Hong-Bae, ein zeitgenössischer Literaturkritiker und Germanistik-Professor, treffend formuliert.

Nicht alle Dichter in Korea schreiben heute wie GIM Nam-Ju. Nicht alle können und sollten schreiben wie er, denn diese Forderung wäre schon wieder ein Diktat, das sich mit der Freiheit des Dichters nicht verträgt. Angesichts von Diktaturen im geteilten Land feindlicher Brüder fordert die Geschichte den koreanischen Dichter aber dazu auf, sich schreibend zu ihr zu verhalten. Gegen drohende Geschichtsvergessenheiten der Gegenwart fordert sie ihn dazu auf, seine eigene Position im Spektrum zwischen harmlosem Dichter, der von der schönen Natur oder von der leidenschaftlichen Liebe schreibt, und todernstem Dichter wie GIM Nam-Ju bewusst einzunehmen.

18) Hong-Bae Lim: Gedichte der Tat und Gewissen des Gedichts, S. 213, in: Yom Mu-Ung / Lim Hong-Bae (Hrsgg.): Die Welt der Literatur von Kim Nam-Ju, Seoul: Changbi 2014, S. $190-214$. 


\section{- Bibliographie}

Choe, In-Hun: Der Platz, in: In-Hun Choe: Sämtliche Werke, Munhakgwajiseongsa 2010, Bd. 1, S. 23-209.

Gim, Nam-Ju: Behausungsort des Gedankens. Gedichte, Seoul: Changjakgwabipyeongsa 1991.

Gim, Won-il: Blaue Seelen. Ein Roman aus sechs Serien-Erzählungen, Seoul: Irum, 2005.

Gim, Won-il: Der Vater des Sohnes. Die Zeit des Vaters und die Kindhiet des Sohns, Seoul: Munhakgwasasangsa 2013.

Han, Sungja: Metapher und Symbol in den Gedichten von Kim Nam-Ju, in: Yom Mu-Ung / Lim Hong-Bae (Hrsgg.): Die Welt der Literatur von Kim Nam-Ju, Seoul: Changbi 2014, S. 235-246.

Hwang, Sok-yong: Der Gast. Roman. Aus dem Koreanischen von Young Lie, Katrin Mensing und Matthias Augustin, München: Deutscher Taschenbuch Verlag 2007.

Hyeon, Gil-Eon: Auf dem Weg nach der Insel, in: Gil-Eon Hyeon: Kann nicht ein jeder zu der Insel gehen? Erzählungen, Seoul: Mulle 2014, S. 111-205.

Lim, Hong-Bae: Gedichte der Tat und Gewissen des Gedichts, in: Yom Mu-Ung / Lim Hong-Bae (Hrsgg.): Die Welt der Literatur von Kim Nam-Ju, Seoul: Changbi 2014, S. 190-214.

Wolf, Christa: Kassandra. Erzählung, Darmstadt und Neuwied 1983.

Yi, Cheong-Jun: Sämtliche Werke, Bd. 4: Die Mauer der Gerüchte, Seoul: Munhakgwajiseongsa 2011. 
국문요약

\section{한국 분단문학의 낙인들로 남은 전쟁}

안 삼 환 (서울대)

한국이 남북으로 분단되고 남북 간에 한국전쟁이 일어난 것은 남북 한국인 사이에 존재하는 어떤 민족지(民族誌)적인 차이점이나 갈등 때문이 아니라, 전적으로 동서 냉전 때문이었다. 따라서, 1950년대 이후의 한국문학에 나타나 는 전쟁은 남북 한국인들 사이의 갈등으로 나타난다기 보다는 냉전시대의 대 리전쟁, 즉 공산주의와 민주주의, 마르크시즘과 기독교의 싸움으로 나타난다. 최인훈의 소설 『광장』에서 주인공 이명준이 남도 아니고 북도 아닌 제3국을 향해 떠날 수밖에 없는 이유도 여기에 있다.

죄없는 양민이 대규모로 살상된 한국전쟁의 실상이 황석영의『손님』(2007), 김원일의『아버지의 아들』(2013) 등 최근에 나온 소설에서야 비로소 온전히 드러나고 있다.

전쟁은 문학작품에서 양민학살 등 전쟁 범죄 자체로만 나타나는 것이 아니 라, 동독의 작가 크리스타 볼프가 그녀의 소설『카산드라』에서도 형상화했듯 이, 권력 및 정보기관이 이적행위를 빙자하여 시인과 작가의 표현권을 탄압하 거나 제한하는 현상으로서도 문제시되며, 시인과 작가들이 이런 탄압과 제한 을 미리 의식하여 자기검열을 하게 되는 것도 전쟁의 한 작용이기도 하다. 이 청준의『소문의 벽』(1971)과 김원일의『푸른 혼』(2005)은 한국문학의 이러한 특수성들을 잘 보여주고 있는 상호보완적인 두 예이다.

특히, 김남주의 시 문학은 우리로 하여금 분단 한국에서 작품을 쓴다는 것 이 무엇을 의미하는 지를 뼈저리게 인식하게 만들고 있다. 그리하여, 그의 시 와 삶은 또한 이러한 분단상황 하에서 작품을 써야 하는 분단 한국의 모든 시인 - 작가로 하여금 자신의 작품이 현재 어떤 좌표 위에서, 무엇을 지향하고 있는 지를 가늠해 볼 수 있게 하는 중요한 지표이기도 하다. 
주제어: 한국전쟁, 분단, 분단문학, 이적행위, 검열, 자기검열

Schlüsselbegriffe: Korea-Krieg, Teilung, Literatur des geteilten

Landes, Feindbegünstigung, Zensur, Selbstzensur

필자 E-mail: samhahn@snu.ac.kr

논문투고일: 2014. 10. 24, 논문심사일: 2014. 11. 18, 게재확정일: 2014. 11. 25. 